\title{
SCIENTIFIC REPORTS

\section{OPEN Prognostic significance of miR-122 expression after curative resection in patients with hepatocellular carcinoma}

Received: 20 August 2018

Accepted: 13 September 2019

Published online: 14 October 2019

\begin{abstract}
Sang Yun $\mathrm{Ha}^{1}$, Jeong IIYu ${ }^{2}$, Changhoon $\mathrm{Choi}^{2}$, So Young Kang ${ }^{1}$, Jae-Won Joh ${ }^{3}$, Seung Woon Paik ${ }^{4}$, Seonwoo Kim ${ }^{5}$, Minji Kim ${ }^{5}$, Hee Chul Park ${ }^{2}$ \& Cheol-Keun Park ${ }^{1,6}$

Downregulation of MicroRNA-122 (miR-122) and its association with cancer progression have been reported in hepatocellular carcinoma (HCC) cell line models and a limited number of HCC samples. Recently, restoration of miR-122 expression by direct delivery of miR-122 yielded promising results in HCCs. However, the prognostic effect of miR-122 expression in human HCC samples is not fully understood. We investigated the expression level of miR-122 by quantitative real-time polymerase chain reaction in 289 curatively resected HCC samples and 20 normal liver samples and evaluated the prognostic effect of miR-122 expression. The relative quantification value of miR-122 was much lower in HCC samples than in normal liver tissues. During a median 119 months of follow-up for survival, the low miR-122 expression group showed shorter recurrence-free survival (RFS) $(p=0.033)$ and intrahepatic recurrence-free survival (IHRFS) $(p=0.014)$, and a trend of short distant metastasis-free survival (DMFS) $(p=0.149)$ than high expression group. On multivariate analysis, miR-122 expression was an independent prognostic factor for RFS, IHRFS and DMFS. Downregulation of miR-122 expression, frequently found in HCC samples, was an independent prognostic factor for RFS after curative resection. Emerging therapeutic approaches targeting miR-122 could be applicable in patients with miR-122 downregulated hepatocellular carcinoma.
\end{abstract}

The overall survival rate of patients with hepatocellular carcinoma (HCC) remains unsatisfactory despite improvements in surveillance and clinical treatment strategies because of frequent recurrence and metastasis even after hepatic resection and lack of effective adjuvant therapy ${ }^{1,2}$. Identification of new therapeutic targets or reliable biomarkers is needed to ensure more effective clinical treatment after curative resection ${ }^{3}$.

MicroRNA-122 (miR-122) is the most abundant liver-specific miRNA, and its expression is conserved in all vertebrates, reflecting its critical role ${ }^{4-7}$. It is well known that miR-122 is involved in lipid metabolism, iron homeostasis, and differentiation of hepatocytes ${ }^{8-12}$. Also, expression of miR-122 has been reported to be downregulated in $\mathrm{HCCs}^{13-20}$, and its role as a tumor suppressant in hepatocellular carcinogenesis has been shown using in vitro and in vivo experimental models ${ }^{10,21,22}$. A few previous studies showed shorter survival time in patients with HCC with low miR-122 expression ${ }^{23-26}$. However, the clinical role of miR-122 expression in human HCC samples is not fully understood.

Based on evidence of the tumor suppressive effect of miR-122 in HCCs, direct delivery of miRNA oligonucleotides into tumors for restoration of miR-122 expression has been performed as a therapeutic strategy against HCC with promising results ${ }^{22,27-29}$. Given the potential clinical application of miR-122 restoration in the treatment of HCCs, it is necessary to evaluate miR-122 expression and its clinical role in HCC samples.

${ }^{1}$ Department of Pathology and Translational Genomics, Samsung Medical Center, Sungkyunkwan University School of Medicine, Seoul, Korea. ${ }^{2}$ Department of Radiation Oncology, Samsung Medical Center, Sungkyunkwan University School of Medicine, Seoul, Korea. ${ }^{3}$ Department of Surgery, Samsung Medical Center, Sungkyunkwan University School of Medicine, Seoul, Korea. ${ }^{4}$ Department of Internal Medicine, Samsung Medical Center, Sungkyunkwan University School of Medicine, Seoul, Korea. ${ }^{5}$ Statistics and Data Center, Samsung Medical Center, Sungkyunkwan University School of Medicine, Seoul, Korea. ${ }^{6}$ Department of Pathology, Anatomic Pathology Reference Lab, Seegene Medical Foundation, Seoul, Korea. Correspondence and requests for materials should be addressed to H.C.P. (email: hee.ro.park@samsung.com) or C.-K.P. (email: ckpark@mf.seegene.com) 
In this study, we examined miR-122 expression by quantitative Reverse-Transcriptase Polymerase Chain Reaction (qRT-PCR) in 289 HCC samples and analyzed the prognostic effect of miR-122 expression in HCC patients with a long-term follow up period (median follow-up 119 months for survival).

\section{Results}

Patients. The characteristics of patients and tumors at the time of surgery are summarized in Table 1 . The median age was 53 years (range, 17-76). Out of 289 patients, 220 (76.1\%) showed HCC related to hepatitis B virus (HBV), and 29 (10.0\%) showed HCC related to hepatitis C virus (HCV). Approximately $80 \%$ of patients had American Joint Committee on Cancer (AJCC) T stage 1 or 2 . Median tumor size was $3.7 \mathrm{~cm}$, and about one-third of the patients had a tumor with larger than $5 \mathrm{~cm}$. Microvascular invasion, major portal invasion, intrahepatic metastasis, and multicentric occurrence was observed in $55.0 \%, 4.5 \%, 23.5 \%$, and $6.6 \%$ of patients, respectively. Approximately 50\% of HCCs occurred in the background of cirrhosis.

miR-122 expression in HCC tissues and its association with clinicopathologic parameters. The assay showed a linear dynamic range of log concentration, and an efficiency of 97\% (Supplementary Fig. S1). The intra- and inter-assay coefficient of variabilities ranged from $0.3 \%$ to $1.8 \%$ and $0.8 \%$ to $1.7 \%$, respectively.

The relative quantification (RQ) value of miR-122 was significantly lower in HCC samples than in normal liver tissues (mean \pm standard deviation: $0.12 \pm 0.37$ vs. $1.07 \pm 1.07, \mathrm{p}<0.001$ by Mann- Whitney U test) (Fig. 1). About $90 \%$ of the HCC samples showed a value lower than the minimum value (0.24) of normal liver tissues, whereas only five HCC cases showed a value higher than the mean value (1.07) of normal liver tissues.

The RQ value of miR-122 showed a tendency to be lower in large sized ( $>5 \mathrm{~cm}$ ) HCCs compared with small $(\leq 5 \mathrm{~cm}) \mathrm{HCCs}$ (mean $0.073 \pm 0.100$ vs. $0.150 \pm 0.445)$ and in BCLC (Barcelona Clinic Liver Cancer) stage B,C compared with BCLC stage 0 ,A (mean $0.076 \pm 0.098$ vs. $0.159 \pm 0.477$ ), but these differences did not reach statistical significance by Mann Whitney test. The RQ value of miR-122 in HCC with other prognostic factors such as advanced AJCC T stage, intrahepatic metastasis, microvascular invasion, or Edmondson grade III or IV was slightly lower than in other groups, but failed to demonstrate statistical significance (data not shown).

The median of RQ value in 289 HCC samples was 0.036 (range, $0.000-4.205$ ). The estimated cutoff value of miR-122 with the highest level of statistical significance related to Recurrence free survival (RFS) was 0.17 by the minimum $\mathrm{p}$-value approach. This cutoff value was internally validated using 1,000 bootstrap samples generated from the study data with replacement. The estimated cutoff value of 0.17 was located between 0.007 and 0.400 , which were the lower and upper 5th percentiles of the estimates, respectively. Out of 289 cases, 44 (15.2\%) were classified into the high expression group and $245(84.8 \%)$ into the low expression group. The associations between miR-122 expression and clinicopathologic parameters are summarized in Table 2. miR-122 expression was not significantly associated with any clinicopathologic factor including well-known prognostic factors of HCC. Clinical information and RQ value of miR-122 in each sample are provided in Supplementary Dataset 1.

Recurrence pattern and predictive factors for early and late intrahepatic recurrence. During the median 120 months (range 2-193) of follow-up, recurrence was detected in 197 patients (68.2\%). Among them, 82 patients $(28.4 \%)$ had both intrahepatic recurrence (IHR) and distant metastasis (DM), 102 patients (35.3\%) had IHR only, and 13 patients (4.5\%) had DM only. Among all patients with IHR (with or without DM), early IHR developed in 125 (43.4\%) patients, and late IHR was detected in 59 of the 145 patients (40.7\%) that were followed for over 2 years.

The results of univariate and multivariate analyses of clinicopathologic parameters and early or late IHR are summarized in Tables 3 and 4. In multivariate analysis, intrahepatic metastasis (HR 8.29 [95\% CI 2.28-30.17], p = 0.001 by the Cox proportional hazard model), Albumin-Bilirubin (ALBI) grade (HR 2.13 [95\% CI 1.10-4.13], $\mathrm{p}=0.026$ by the Cox proportional hazard model), and liver cirrhosis (HR 2.01 [95\% CI 1.08-3.72], $\mathrm{p}=0.028$ by the Cox proportional hazard model) were independent predictive factors for early IHR. miR-122 expression showed marginal significance for predicting early IHR (HR 2.02 [95\% CI 0.91-4.47], $\mathrm{p}=0.083$ by the Cox proportional hazard model).

For predicting late IHR, etiology and miR-122 expression were significant factors in univariate analysis, and miR-122 expression was the only significant factor in multivariate analysis (HR 3.04 [95\% CI 1.02-9.06], $\mathrm{p}=0.046$ by the Cox proportional hazard model).

Impact of miR-122 expression on the recurrence-free survival of HCC patients. Patients with low miR-122 expression showed shorter IHRFS and RFS rates than those with high miR-122 expression $(\mathrm{p}=0.014$ and $\mathrm{p}=0.033$ by log-rank test, respectively) and a trend toward a shorter DMFS rate ( $\mathrm{p}=0.149$ by log-rank test) (Fig. 2). Results of univariate analysis of probable prognostic factors for intrahepatic RFS, DMFS, and RFS are summarized in Table 5. In multivariate analyses, significant prognostic factors were intrahepatic metastasis, multicentric occurrence, ALBI grade, and miR-122 expression for intrahepatic RFS; Edmondson grade, intrahepatic metastasis, and miR-122 expression for DMFS; and intrahepatic metastasis, ALBI grade, and MiR-122 expression for RFS (Table 6). In addition to intrahepatic metastasis, miR-122 expression was an independent prognostic factor for IHRFS (HR 1.89 [95\% CI 1.16-3.07], $\mathrm{p}=0.010$ by the time-dependent Cox model), DMFS (HR 2.14 [95\% CI 1.05-4.36], $\mathrm{p}=0.036$ by the time-dependent Cox model), and RFS (HR 2.17 [95\% CI 1.34-3.52], $\mathrm{p}=0.002$ by the time-dependent Cox model).

\section{Discussion}

In this study, we demonstrated that miR-122 expression was downregulated in 289 HCC samples compared with normal liver tissues and was an independent predictive factor for late IHR as well as an independent prognostic factor for RFS regardless of recurrence pattern (intrahepatic recurrence or distant metastasis) in a large cohort of HCC patients with long-term follow-up. 


\begin{tabular}{|c|c|c|}
\hline Characteristics & & No. of patients (\%) \\
\hline Age & Median (range) & $53(17-76)$ \\
\hline \multirow{2}{*}{ Sex } & Female & $51(17.6)$ \\
\hline & Male & $238(82.4)$ \\
\hline \multirow{5}{*}{ Etiology } & Hepatitis B virus & $220(76.1)$ \\
\hline & Hepatitis $\mathrm{C}$ virus & $25(8.6)$ \\
\hline & Hepatitis $B$ virus and Hepatitic $C$ virus & $4(1.4)$ \\
\hline & Alcohol & $18(6.2)$ \\
\hline & Others & $22(7.6)$ \\
\hline \multirow{2}{*}{ Child-Pugh Class } & A & $288(99.7)$ \\
\hline & B & $1(0.3)$ \\
\hline \multirow{3}{*}{ Albumin level, g/dL } & Median (range) & $4.0(2.8-5.0)$ \\
\hline & $>3.5$ & $260(90.0)$ \\
\hline & $\leq 3.5$ & $29(10.0)$ \\
\hline \multirow{2}{*}{ ALBI grade } & 2 & $93(32.2)$ \\
\hline & 1 & $196(67.8)$ \\
\hline \multirow{3}{*}{ AFP level, ng/mL } & Median (range) & $169.5(1.0-1667054.0)$ \\
\hline & $\leq 200$ & $175(62.7)$ \\
\hline & $>200$ & $104(37.3)$ \\
\hline \multirow{5}{*}{ AJCC T-stage } & 1 & $122(42.2)$ \\
\hline & 2 & $116(40.1)$ \\
\hline & $3 \mathrm{~A}$ & $33(11.4)$ \\
\hline & $3 \mathrm{~B}$ & $12(4.2)$ \\
\hline & 4 & $6(2.1)$ \\
\hline \multirow{6}{*}{ BCLC stage } & 0 & $2(0.7)$ \\
\hline & $\mathrm{A} 1$ & $142(49.1)$ \\
\hline & A2 & $5(1.7)$ \\
\hline & A4 & $16(5.5)$ \\
\hline & $\mathrm{B}$ & $109(37.7)$ \\
\hline & $\mathrm{C}$ & $15(5.2)$ \\
\hline \multirow{3}{*}{ Tumor size, cm } & Median (range) & $3.7(1.0-21.0)$ \\
\hline & $\leq 5.0$ & $191(66.1)$ \\
\hline & $>5.0$ & $98(33.9)$ \\
\hline \multirow{3}{*}{ Edmondson grade } & I & $32(11.1)$ \\
\hline & II & $233(80.6)$ \\
\hline & III & $24(8.3)$ \\
\hline \multirow{2}{*}{ Microvascular invasion } & Yes & $159(55.0)$ \\
\hline & No & $130(45.0)$ \\
\hline \multirow{2}{*}{ Major portal vein invasion } & Yes & $13(4.5)$ \\
\hline & No & $276(95.5)$ \\
\hline \multirow{2}{*}{ Intrahepatic metastasis } & Yes & $68(23.5)$ \\
\hline & No & $221(76.5)$ \\
\hline \multirow{2}{*}{ Multicentric occurrence } & Yes & $19(6.6)$ \\
\hline & No & $270(93.4)$ \\
\hline \multirow{6}{*}{ Backgound liver tissue } & Cirrhosis & $145(50.2)$ \\
\hline & Chronic active hepatitis & $67(23.2)$ \\
\hline & Chronic persistent hepatitis & $44(15.2)$ \\
\hline & Alcoholic hepatitis & $15(5.2)$ \\
\hline & Reactive hepatitis & $13(4.5)$ \\
\hline & Others & $5(1.7)$ \\
\hline
\end{tabular}

Table 1. Characteristics of patients and tumors, at the time of surgery. ALBI, Albumin-Bilirubin; AFP, $\alpha$-fetoprotein; AJCC, American Joint Committee on Cancer; BCLC, Barcelona Clinic Liver Cancer; ${ }^{\text {a) Partial }}$ data was not available.

There have been numerous reports on the role of miR-122 as a tumor suppressor in hepatocarcinogenesis, performed at various confidence levels. Downregulation of miR-122 in HCCs has been reported in several studies ${ }^{13-20}$, leading to further in vitro and in vivo studies. Tsai et al. showed that miR-122 was significantly downregulated in T3 HCCs with intrahepatic metastasis compared with T1 HCCs or adjacent normal liver tissue, and that restoration of miR-122 reduced in vitro migration, invasion, and anchorage-independent growth as well as tumorigenesis 


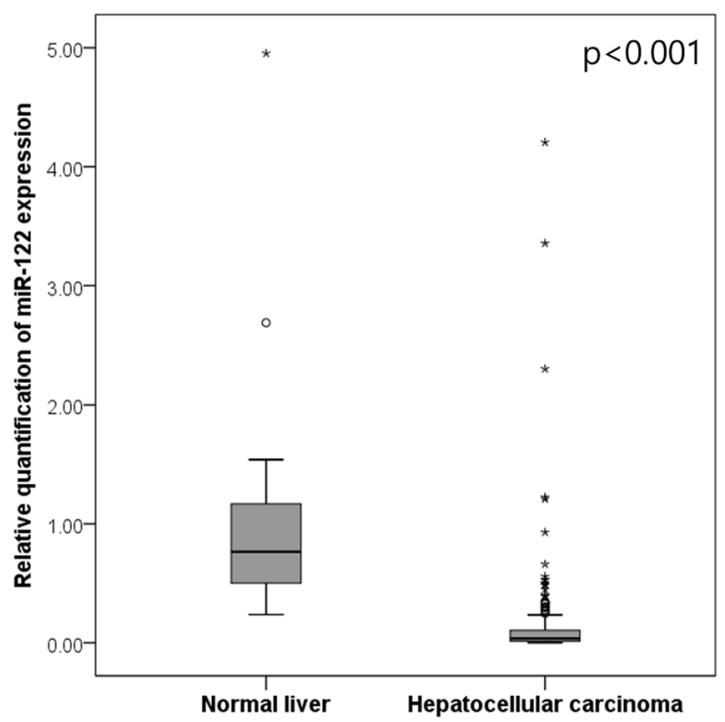

Figure 1. The relative quantification value of miR-122 in hepatocellular carcinoma and normal liver.

in vivo in a nude mouse xenograft model $^{21}$. In 2012, Hsu et al. and Tsai et al. provided the strongest evidence for an antitumor function of miR-122 in liver. In their studies, mice with germline knockout or liver-specific knockout of miR-122 developed spontaneous HCCs in addition to steatohepatitis and fibrosis, and restoration of miR-122 reduced tumor development ${ }^{10,22}$. However, the clinical effect of miR-122 expression in HCC patients, especially on patient survival, has not been fully elucidated. Coulouarn et al. reported that patients with low miR-122 expression showed shorter overall survival times than those with high miR-122 expression ( $30.3 \pm 8.0$ months vs. $83.7 \pm 10.3$ months, $\mathrm{p}<0.001$ ) in 64 HCC patients ${ }^{26}$. They also showed that miR-122 expression was low in HCCs with poor differentiation, high proliferation, low apoptotic index, and large tumor size ${ }^{26}$. In a recent meta-analysis by Zhang et al. incorporating of 4 studies containing 328 patients, low miR-122 expression in tissue or fine needle aspiration sample was significantly associated with unfavorable overall survival and progression free survival. In validation using TCGA dataset, low miR-122 expression was significantly associated with OS and marginally associated with $\mathrm{PFS}^{30}$. Also, significance of serum miR-122 expression in HCC have been reported. Qiao et al. showed that serum miR-122 expression was significantly lower in HBV-related HCC patients than in benign liver disease group or control group ${ }^{31}$. A few recent reports suggested serum exosomal miR-122 as a predictive biomarker in transarterial chemoembolization-treated HCC patients or diagnostic biomarkers for $\mathrm{HCC}^{32,33}$.

In this study, we showed that miR-122 expression was downregulated in 289 HCC samples compared with normal liver tissues. About $90 \%$ of the HCC samples showed a value lower than the minimum expression value of normal liver tissues. Also, we demonstrated that miR-122 expression was an independent prognostic factor for RFS in a large cohort of 289 HCC patients with long-term follow-up. These results are consistent with results from previous studies and provide clinical evidence suggesting miR-122 as a potential therapeutic tool targeting HCC. Interestingly, survival curves are clearly distinguished after 2 years from surgery, as depicted in survival curve of Fig. 2, and low expression of miR-122 was associated with frequent late intrahepatic recurrence, which could represent a de novo HCC, not metastasis from primary tumor. Based on these results, we can suggest that patients with HCC having low expression of miR-122 may require intensive follow-up even after 2 years from surgery. These results are somewhat different from previous studies showing association between low expression of miR-122 and aggressive tumor behavior which represents early intrahepatic recurrence ${ }^{22,26,34}$. This study includes HCCs with curative resection, which had no known metastasis at the time of surgery. We observed that miR-122 expression is slightly lower in HCCs with factors associated with tumor aggressiveness, such as larger size $(>5 \mathrm{~cm})$, intrahepatic metastasis, higher Edmondson grade, or microvascular invasion, but it did not reach the statistical significance. The effect could be less obvious because of the relatively uniform study population. Meanwhile, miR-122 down-regulation is known to be involved in the progression of liver fibrosis and emergence of de novo $\mathrm{HCC}^{10}$. It can be inferred that de-novo HCCs by down-regulation of miR-122 could have low expression of miR-122. There have been only 4 studies regarding prognostic effect of miR-122 expression in HCC tissue samples, and the largest patient number was $144^{23-26}$. Further study is necessary for validating the prognostic effect of miR-122 expression.

Based on previous studies supporting a tumor suppressive effect of miR-122 in HCCs, restoration of miR-122 expression represents an interesting strategy for the treatment of HCC. Direct delivery of miRNA mimic oligonucleotides into tumors by viral vectors such as the adeno-associated virus 8 (AAV8) serotype or LNP-DP1, a cationic lipid- based nanoparticle, has been tested with promising results ${ }^{22,27-29}$. It is noteworthy that the first miRNA mimic, MRX34, a liposomal miRNA 34 mimic, is now undergoing testing in a multicenter phase I study in a variety of cancers including HCCs (ClinicalTrials.gov.identifier: NCT01829971A) ${ }^{35}$. In addition, miR-122 has therapeutic applications in the aspect of sensitization of chemotherapy. It has been reported that restoration of miR-122 sensitizes HCCs to chemotherapeutic agents such as sorafenib, doxorubicin, vincristine, and cisplatin ${ }^{36-39}$. A combination of miR-122 restoration and chemotherapy could be another therapeutic strategy against HCC. 


\begin{tabular}{|c|c|c|c|c|}
\hline & \multirow[b]{3}{*}{ Total } & \multicolumn{2}{|c|}{ miR122 expression } & \multirow[b]{3}{*}{ p value } \\
\hline & & Low $(\mathrm{RQ}$ value $\leq 0.17)$ & High (RQ value $>0.17$ ) & \\
\hline & & $\mathrm{n}=245(24.8 \%)$ & $\mathrm{n}=44(15.2 \%)$ & \\
\hline \multicolumn{5}{|l|}{ Age, year } \\
\hline$\leq 55$ & 167 & $143(58.4 \%)$ & $24(54.5 \%)$ & 0.636 \\
\hline$>55$ & 122 & $102(41.6 \%)$ & $20(45.5 \%)$ & \\
\hline \multicolumn{5}{|l|}{ Gender } \\
\hline Female & 51 & $45(18.4 \%)$ & $6(13.6 \%)$ & 0.448 \\
\hline Male & 238 & $200(81.6 \%)$ & $38(86.4 \%)$ & \\
\hline \multicolumn{5}{|l|}{ Tumor size, cm } \\
\hline$\leq 5.0$ & 191 & $160(65.3 \%)$ & $31(70.5 \%)$ & 0.507 \\
\hline$>5.0$ & 98 & $85(34.7 \%)$ & $13(29.5 \%)$ & \\
\hline \multicolumn{5}{|c|}{ Edmondson grade } \\
\hline I & 32 & $27(11.0 \%)$ & $5(11.4 \%)$ & $0.617^{\mathrm{c})}$ \\
\hline II & 233 & $199(81.2 \%)$ & $34(77.3 \%)$ & \\
\hline III & 24 & $19(7.8 \%)$ & $5(11.4 \%)$ & \\
\hline \multicolumn{5}{|c|}{ Microvascular invasion } \\
\hline$(-)$ & 130 & $110(44.9 \%)$ & $20(45.5 \%)$ & 0.946 \\
\hline$(+)$ & 159 & $135(55.1 \%)$ & $24(54.5 \%)$ & \\
\hline \multicolumn{5}{|c|}{ Major portal vein invasion } \\
\hline$(-)$ & 276 & $233(95.1 \%)$ & $43(97.7 \%)$ & 0.439 \\
\hline$(+)$ & 13 & $12(4.9 \%)$ & $1(2.3 \%)$ & \\
\hline \multicolumn{5}{|c|}{ Intrahepatic metastasis } \\
\hline$(-)$ & 221 & $187(76.3 \%)$ & $34(77.3 \%)$ & 0.892 \\
\hline$(+)$ & 68 & $58(23.7 \%)$ & $10(22.8 \%)$ & \\
\hline \multicolumn{5}{|c|}{ Multicentric occurrence } \\
\hline$(-)$ & 270 & $230(93.9 \%)$ & $40(90.1 \%)$ & $0.506^{c)}$ \\
\hline$(+)$ & 19 & $15(6.1 \%)$ & $4(9.9 \%)$ & \\
\hline \multicolumn{5}{|l|}{ AJCC T-stage } \\
\hline 1 & 122 & $105(42.9 \%)$ & $17(38.6 \%)$ & $0.942^{\mathrm{c})}$ \\
\hline 2 & 116 & $97(39.6 \%)$ & $19(43.2 \%)$ & \\
\hline 3 & 45 & $38(18.4 \%)$ & $7(15.9 \%)$ & \\
\hline 4 & 6 & $5(2.0 \%)$ & $1(2.3 \%)$ & \\
\hline \multicolumn{5}{|l|}{ BCLC stage } \\
\hline $0-\mathrm{A}$ & 165 & $138(56.3 \%)$ & $27(61.4 \%)$ & $0.887^{\mathrm{c})}$ \\
\hline $\mathrm{B}$ & 109 & $94(44.5 \%)$ & $15(34.1 \%)$ & \\
\hline $\mathrm{C}$ & 15 & $13(5.3 \%)$ & $2(4.5 \%)$ & \\
\hline \multicolumn{5}{|c|}{ Albumin level, $\mathrm{g} / \mathrm{dL}$} \\
\hline$>3.5$ & 260 & $218(89.0 \%)$ & $42(93.3 \%)$ & $0.276^{\mathrm{c})}$ \\
\hline$\leq 3.5$ & 29 & $27(11.0 \%)$ & $2(6.7 \%)$ & \\
\hline \multicolumn{5}{|c|}{ AFP level, $\mathbf{n g} / \mathrm{mL}^{\mathrm{a})}$} \\
\hline$\leq 200$ & 175 & $146(61.1 \%)$ & $29(72.5 \%)$ & 0.167 \\
\hline$>200$ & 104 & $93(38.9 \%)$ & $11(27.5 \%)$ & \\
\hline \multicolumn{5}{|l|}{ Etiology } \\
\hline Non-viral & 40 & $33(16.3 \%)$ & $7(15.9 \%)$ & $0.567^{\mathrm{c})}$ \\
\hline HBV & 220 & $186(89.8 \%)$ & $34(77.3 \%)$ & \\
\hline $\mathrm{HCV}$ & 25 & $23(9.4 \%)$ & $2(4.5 \%)$ & \\
\hline $\mathrm{HBV}$ and $\mathrm{HCV}$ & 4 & $3(1.2 \%)$ & $1(2.3 \%)$ & \\
\hline \multicolumn{5}{|l|}{ Liver cirrhosis } \\
\hline$(-)$ & 144 & $125(51.0 \%)$ & $19(43.2 \%)$ & 0.338 \\
\hline$(+)$ & 145 & $120(49.0 \%)$ & $25(56.8 \%)$ & \\
\hline \multicolumn{5}{|c|}{ Early intrahepatic recurrence } \\
\hline ( $\leq 2$ years $)$ & & & & \\
\hline$(-)^{\mathrm{b})}$ & 164 & $134(54.7 \%)$ & $30(68.2 \%)$ & 0.096 \\
\hline$(+)$ & 125 & $111(45.3 \%)$ & $14(31.8 \%)$ & \\
\hline Late intrahepat & & & & \\
\hline ( $>2$ years $)$ & & & & \\
\hline Continued & & & & \\
\hline
\end{tabular}




\begin{tabular}{|c|c|c|c|c|}
\hline & \multirow[b]{3}{*}{ Total } & \multicolumn{2}{|l|}{ miR122 expression } & \multirow[b]{3}{*}{ p value } \\
\hline & & Low $(\mathrm{RQ}$ value $\leq 0.17)$ & High $($ RQ value $>0.17)$ & \\
\hline & & $\mathrm{n}=245(24.8 \%)$ & $\mathrm{n}=44(15.2 \%)$ & \\
\hline$(-)^{\mathrm{b})}$ & 85 & $65(55.1 \%)$ & $20(76.9 \%)$ & 0.040 \\
\hline$(+)$ & 59 & $53(44.9 \%)$ & $6(23.1 \%)$ & \\
\hline \multicolumn{5}{|c|}{ Distant metastasis } \\
\hline$(-)$ & 194 & $160(65.3 \%)$ & $34(77.3 \%)$ & 0.120 \\
\hline$(+)$ & 95 & $85(34.7 \%)$ & $10(22.7 \%)$ & \\
\hline
\end{tabular}

Table 2. The association between miR122 expression and clinicopathologic parameters. AJCC, American Joint Committee on Cancer; BCLC, Barcelona Clinic Liver Cancer; AFP, $\alpha$-fetoprotein; HBV, hepatitis B virus; HCV, hepatitis $\mathrm{C}$ virus. ${ }^{\text {a) }}$ Partial data was not available ${ }^{\mathrm{b})}$ No early or late recurrence, ${ }^{\mathrm{c}}$ by Fisher's exact test, otherwise by chi-square test.

\begin{tabular}{|c|c|c|c|c|}
\hline & \multicolumn{2}{|l|}{ Univariate } & \multicolumn{2}{|l|}{ Multivariate } \\
\hline & HR (95\% CI) & p value & HR (95\% CI) & p value \\
\hline \multicolumn{5}{|l|}{ Age, year } \\
\hline$>55$ vs $\leq 55$ & $1.01(0.63-1.62)$ & 0.956 & & \\
\hline \multicolumn{5}{|l|}{ Gender } \\
\hline Male vs Female & $1.11(0.60-2.05)$ & 0.742 & & \\
\hline \multicolumn{5}{|l|}{ Tumor size, cm } \\
\hline$>5.0 \mathrm{vs} \leq 5.0$ & $1.95(1.19-3.19)$ & 0.008 & $0.76(0.21-2.77)$ & 0.681 \\
\hline \multicolumn{5}{|l|}{ Edmondson grade } \\
\hline III vs I, II & $1.94(0.83-4.53)$ & 0.125 & $1.16(0.42-3.20)$ & 0.78 \\
\hline \multicolumn{5}{|c|}{ Microvascular invasion } \\
\hline$(+)$ vs $(-)$ & $3.12(1.90-5.10)$ & $<0.001$ & $1.49(0.77-2.88)$ & 0.238 \\
\hline \multicolumn{5}{|c|}{ Major portal vein invasion } \\
\hline$(+)$ vs $(-)$ & $4.67(1.26-17.33)$ & 0.021 & $0.57(0.16-2.86)$ & 0.498 \\
\hline \multicolumn{5}{|c|}{ Intrahepatic metastasis } \\
\hline$(+)$ vs $(-)$ & $10.28(5.18-20.40)$ & $<0.001$ & $8.29(2.28-30.17)$ & 0.001 \\
\hline \multicolumn{5}{|c|}{ Multicentric occurrence } \\
\hline$(+)$ vs $(-)$ & $0.95(0.37-2.44)$ & 0.917 & & \\
\hline \multicolumn{5}{|l|}{ AJCC T-stage } \\
\hline 3,4 vs 1,2 & $5.74(2.86-11.56)$ & $<0.001$ & $1.08(0.25-4.67)$ & 0.924 \\
\hline \multicolumn{5}{|l|}{ BCLC stage } \\
\hline $\mathrm{B}, \mathrm{C}$ vs $0, \mathrm{~A}$ & $2.93(1.80-4.75)$ & $<0.001$ & $1.48(0.44-5.01)$ & 0.527 \\
\hline \multicolumn{5}{|l|}{ Albumin level, g/dL } \\
\hline$\leq 3.5 \mathrm{vs}>3.5$ & $1.99(0.92-4.35)$ & 0.083 & $0.97(0.35-2.66)$ & 0.948 \\
\hline \multicolumn{5}{|l|}{ ALBI grade } \\
\hline 2 vs 1 & $2.79(1.68-4.63)$ & $<0.001$ & $2.13(1.10-4.13)$ & 0.026 \\
\hline \multicolumn{5}{|l|}{ AFP level, ng/mL } \\
\hline$>200$ vs $\leq 200$ & $1.36(0.83-2.21)$ & 0.222 & & \\
\hline \multicolumn{5}{|l|}{ Etiology } \\
\hline Viral vs Non-viral & $2.23(1.07-4.65)$ & 0.033 & $1.13(0.48-2.67)$ & 0.777 \\
\hline \multicolumn{5}{|l|}{ Liver cirrhosis } \\
\hline$(+)$ vs $(-)$ & $1.79(1.12-2.87)$ & 0.015 & $2.01(1.08-3.72)$ & 0.028 \\
\hline \multicolumn{5}{|l|}{ miR122 expression } \\
\hline Low vs High & $1.78(0.90-3.51)$ & 0.099 & $2.02(0.91-4.47)$ & 0.083 \\
\hline
\end{tabular}

Table 3. Univariate and multivariate analyses for predicting early intrahepatic recurrence. AJCC, American Joint Committee on Cancer; BCLC, Barcelona Clinic Liver Cancer; ALBI, Albumin-Bilirubin; AFP, $\alpha$-fetoprotein.

This study has some limitations. First, this study was retrospectively performed in a single institution and included patients who had undergone curative resection for primary tumor, thus possibly introducing unavoidable selection bias. Consequently, our results should be interpreted cautiously, and further study with a large population and prospective design is needed. Second, the estimated cutoff value of miR-122 expression was not validated in an independent cohort. We redeemed this by internal validation using 1,000 bootstrap samples generated from the study data with replacement, but further external validation would be required. 


\begin{tabular}{|c|c|c|c|c|}
\hline & \multicolumn{2}{|l|}{ Univariate } & \multicolumn{2}{|l|}{ Multivariate } \\
\hline & HR (95\% CI) & p value & HR (95\% CI) & p value \\
\hline \multicolumn{5}{|l|}{ Age, year } \\
\hline$>55$ vs $\leq 55$ & $1.08(0.55-2.13)$ & 0.823 & & \\
\hline \multicolumn{5}{|l|}{ Gender } \\
\hline Male vs Female & $1.23(0.52-2.90)$ & 0.645 & & \\
\hline \multicolumn{5}{|l|}{ Tumor size, $\mathrm{cm}$} \\
\hline$>5.0 \mathrm{vs} \leq 5.0$ & $0.52(0.24-1.13)$ & 0.100 & $0.59(0.24-1.41)$ & 0.232 \\
\hline \multicolumn{5}{|l|}{ Edmondson grade } \\
\hline III vs I, II & $0.71(0.17-2.94)$ & 0.632 & & \\
\hline \multicolumn{5}{|c|}{ Microvascular invasion } \\
\hline$(+) \mathrm{vs}(-)$ & $0.74(0.37-1.45)$ & 0.375 & & \\
\hline \multicolumn{5}{|c|}{ Major portal vein invasion } \\
\hline$(+) \mathrm{vs}(-)$ & NA & & & \\
\hline \multicolumn{5}{|c|}{ Intrahepatic metastasis } \\
\hline$(+) \mathrm{vs}(-)$ & $1.09(0.23-5.04)$ & 0.917 & & \\
\hline \multicolumn{5}{|c|}{ Multicentric occurrence } \\
\hline$(+) \mathrm{vs}(-)$ & $6.11(0.67-56.11)$ & 0.110 & $4.08(0.42-39.65)$ & 0.225 \\
\hline \multicolumn{5}{|l|}{ AJCC T-stage } \\
\hline 3,4 vs 1,2 & $0.86(0.20-3.73)$ & 0.837 & & \\
\hline \multicolumn{5}{|l|}{ 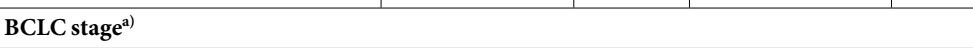 } \\
\hline $\mathrm{B}, \mathrm{C}$ vs $0, \mathrm{~A}$ & $0.52(0.24-1.11)$ & 0.090 & & \\
\hline \multicolumn{5}{|c|}{ Albumin level, g/dL } \\
\hline$\leq 3.5$ vs $>3.5$ & $0.68(0.16-2.83)$ & 0.595 & & \\
\hline \multicolumn{5}{|l|}{ ALBI grade } \\
\hline 2 vs 1 & $1.22(0.54-2.77)$ & 0.637 & & \\
\hline \multicolumn{5}{|l|}{ AFP level, ng/mL } \\
\hline$>200$ vs $\leq 200$ & $1.84(0.91-3.75)$ & 0.092 & $1.86(0.88-3.95)$ & 0.107 \\
\hline \multicolumn{5}{|l|}{ Etiology } \\
\hline Viral vs Non-viral & $2.72(1.02-7.26)$ & 0.046 & $1.86(0.65-5.33)$ & 0.246 \\
\hline \multicolumn{5}{|l|}{ Liver cirrhosis } \\
\hline$(+) v s(-)$ & $1.75(0.89-3.42)$ & 0.104 & $1.43(0.66-3.10)$ & 0.366 \\
\hline \multicolumn{5}{|l|}{ miR122 expression } \\
\hline Low vs High & $2.72(1.02-7.26)$ & 0.046 & $3.04(1.02-9.06)$ & 0.046 \\
\hline
\end{tabular}

Table 4. Univariate and multivariate analyses for predicting late intrahepatic recurrence. AJCC, American Joint

Committee on Cancer; BCLC, Barcelona Clinic Liver Cancer; ALBI, Albumin-Bilirubin; AFP, $\alpha$-fetoprotein.

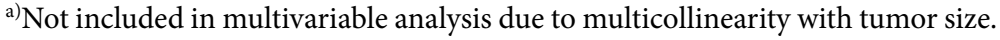

A

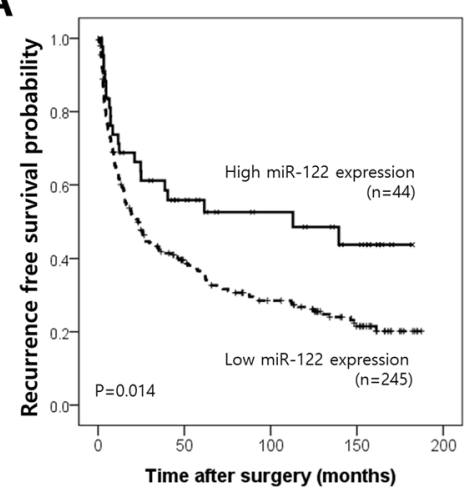

B

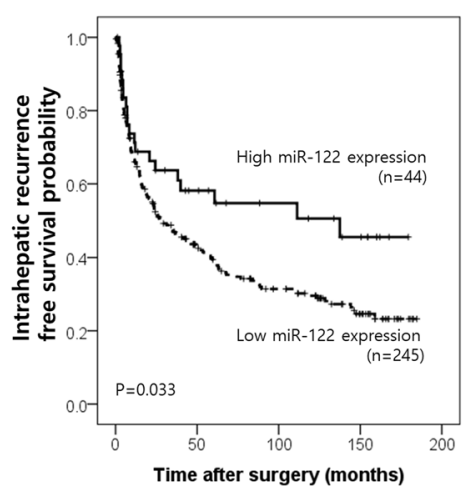

C

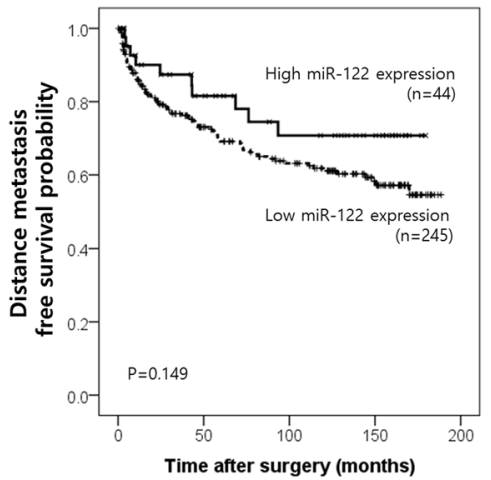

Figure 2. Kaplan Meier survival curves of recurrence free survival (A), intrahepatic recurrence free survival (B) and distant metastasis free survival (C) according to miR-122 expression status. 


\begin{tabular}{|c|c|c|c|c|c|c|}
\hline & \multicolumn{2}{|c|}{ Intrahepatic recurrence free survival } & \multicolumn{2}{|c|}{ Distant metastasis free survival } & \multicolumn{2}{|c|}{ Recurrence free survival } \\
\hline & HR $(95 \% \mathrm{CI})$ & p value & HR $(95 \%$ CI $)$ & p value & HR $(95 \% \mathrm{CI})$ & p value \\
\hline \multicolumn{7}{|l|}{ Age, year } \\
\hline$>55$ vs $\leq 55$ & $1.04(0.78-1.40)$ & 0.791 & $1.00(0.67-1.51)$ & 0.995 & $0.96(0.72-1.27)$ & 0.756 \\
\hline \multicolumn{7}{|l|}{ Gender } \\
\hline Male vs Female & $1.15(0.78-1.70)$ & 0.469 & $0.81(0.50-1.30)$ & 0.38 & $1.06(0.74-1.53)$ & 0.754 \\
\hline \multicolumn{7}{|l|}{ Tumor size, cm } \\
\hline$>5.0 \mathrm{vs} \leq 5.0$ & $1.43(1.05-1.93)$ & 0.022 & $2.37(1.58-3.55)$ & $<0.001$ & $1.68(1.26-2.24)$ & $<0.001$ \\
\hline \multicolumn{7}{|l|}{ Edmondson grade } \\
\hline III vs I, II & $1.51(0.92-2.49)$ & 0.107 & $2.88(1.63-5.09)$ & $<0.001$ & $1.81(1.13-2.90)$ & 0.014 \\
\hline \multicolumn{7}{|c|}{ Microvascular invasion } \\
\hline$(+) \mathrm{vs}(-)$ & $1.86(1.39-2.50)$ & $<0.001$ & $2.85(1.83-4.44)$ & $<0.001$ & $2.14(1.60-2.86)$ & $<0.001$ \\
\hline \multicolumn{7}{|c|}{ Major portal vein invasion } \\
\hline$(+) \mathrm{vs}(-)$ & $3.31(1.74-6.29)$ & $<0.001$ & $6.02(3.09-11.73)$ & $<0.001$ & $3.43(1.86-6.33)$ & $<0.001$ \\
\hline \multicolumn{7}{|c|}{ Intrahepatic metastasis } \\
\hline$(+) \mathrm{vs}(-)$ & $4.67(3.37-6.47)$ & $<0.001$ & $4.76(3.12-7.28)$ & $<0.001$ & $4.58(3.34-6.29)$ & $<0.001$ \\
\hline \multicolumn{7}{|c|}{ Multicentric occurrence } \\
\hline$(+) \mathrm{vs}(-)$ & $1.57(0.87-2.82)$ & 0.136 & $1.17(0.51-2.67)$ & 0.713 & $1.39(0.78-2.51)$ & 0.268 \\
\hline \multicolumn{7}{|l|}{ AJCC T-stage } \\
\hline 3,4 vs 1,2 & $3.21(2.26-4.57)$ & $<0.001$ & $4.57(2.94-7.12)$ & $<0.001$ & $3.84(2.73-5.39)$ & $<0.001$ \\
\hline \multicolumn{7}{|l|}{ BCLC stage } \\
\hline $\mathrm{B}, \mathrm{C}$ vs $0, \mathrm{~A}$ & $1.82(1.36-2.43)$ & $<0.001$ & $2.74(1.82-4.12)$ & $<0.001$ & $2.05(1.54-2.71)$ & $<0.001$ \\
\hline \multicolumn{7}{|c|}{ Albumin level, $\mathrm{g} / \mathrm{dL}$} \\
\hline$\leq 3.5$ vs $>3.5$ & $1.92(1.21-3.03)$ & 0.005 & $1.80(0.98-3.30)$ & 0.058 & $1.84(1.18-2.87)$ & 0.008 \\
\hline \multicolumn{7}{|l|}{ ALBI grade } \\
\hline 2 vs 1 & $1.82(1.35-2.47)$ & $<0.001$ & $1.60(1.06-2.43)$ & 0.026 & $1.85(1.38-2.47)$ & $<0.001$ \\
\hline \multicolumn{7}{|l|}{ AFP level, ng/mL } \\
\hline$>200$ vs $\leq 200$ & $1.42(1.05-1.91)$ & 0.023 & $1.76(1.17-2.66)$ & 0.007 & $1.62(1.21-2.16)$ & 0.001 \\
\hline \multicolumn{7}{|l|}{ Etiology } \\
\hline Viral vs Non-viral & $1.90(1.15-3.13)$ & 0.012 & $1.60(0.80-3.17)$ & 0.182 & $1.88(1.17-3.02)$ & 0.009 \\
\hline \multicolumn{7}{|l|}{ Liver cirrhosis } \\
\hline$(+) \mathrm{vs}(-)$ & $1.43(1.07-1.92)$ & 0.015 & $1.05(0.70-1.57)$ & 0.826 & $1.34(1.02-1.78)$ & 0.039 \\
\hline \multicolumn{7}{|l|}{ miR122 expression } \\
\hline Low vs High & $1.65(1.04-2.63)$ & 0.035 & $1.61(0.84-3.11)$ & 0.153 & $1.75(1.11-2.75)$ & 0.016 \\
\hline
\end{tabular}

Table 5. Univariate analysis of probable prognostic factors in intrahepatic recurrence- free survival, distant metastasis- free survival, and recurrence free survival. AJCC, American Joint Committee on Cancer; BCLC, Barcelona Clinic Liver Cancer; ALBI, Albumin-Bilirubin; AFP, $\alpha$-fetoprotein.

Despite these limitations, our study provides valuable information confirming the prognostic significance of miR-122 expression in the largest cohort of HCC patients with long-term follow-up. Our data showed that miR122 is an independent predictive factor for late IHR and an independent prognostic factor for RFS regardless of recurrence pattern, either intrahepatic recurrence or distant metastasis.

In conclusion, miR-122 expression is frequently downregulated in HCCs, and miR-122 expression is an independent prognostic factor for recurrence-free survival after curative resection. Emerging therapeutic approaches targeting miR-122 could be applicable in patients with miR-122-downregulated hepatocellular carcinoma.

\section{Material and Methods}

Patients and samples. Initially, a total of 291 patients who underwent curative resection for primary HCC at Samsung Medical Center, Seoul, Korea, from July 2000 to May 2006 were enrolled. This cohort is the same as the previous study of our group ${ }^{40}$, and this study has been performed independently of previous study. Samples from 2 patients had poor RNA quality and experiments were failed in these samples. Finally, analyses were performed in remaining 289 patients. Curative resection was defined as complete resection of all tumor nodules without involvement of microscopic resection margins and no detected residual tumor on computed tomography scans at 1 month after surgery. Twenty normal liver samples, which were confirmed biochemically and histologically and mostly obtained from patients with liver resection due to metastatic colorectal cancer, were selected as normal controls. The Institutional Review Board of Samsung Medical Center approved this study (2016-11-098), and waived informed consent. All research was performed in accordance with relevant guidelines/regulations.

By reviewing the medical records, clinical parameters such as age, gender, date of surgery, serum $\alpha$-fetoprotein (AFP), and serum albumin were collected. HCC was diagnosed by basically histological feature by HE slide which shows hepatocytic differentiation, occasionally with aid of immunohistochemical staining of Hepatocyte antigen, AFP, or cytokeratin 19 when differentiation is poor. Histopathologic features of HCCs, including tumor 


\begin{tabular}{|c|c|c|c|c|c|c|}
\hline & \multicolumn{2}{|c|}{ Intrahepatic recurrence free survival } & \multicolumn{2}{|c|}{ Distant metastasis free survival } & \multicolumn{2}{|c|}{ Recurrence free survival } \\
\hline & HR $(95 \% \mathrm{CI})$ & p value & HR $(95 \% \mathrm{CI})$ & \begin{tabular}{|l|l}
$p$ value \\
\end{tabular} & HR $(95 \% \mathrm{CI})$ & p value \\
\hline \multicolumn{7}{|l|}{ Tumor size, cm } \\
\hline$>5.0 \mathrm{vs} \leq 5.0$ & $0.97(0.44-2.13)$ & 0.935 & $1.05(0.39-2.83)$ & 0.929 & $0.94(0.60-1.95)$ & 0.788 \\
\hline \multicolumn{7}{|l|}{ Edmondson grade } \\
\hline III vs I, II & $1.12(0.6401 .94)$ & 0.699 & $2.47(1.34-4.56)$ & 0.004 & $1.22(0.73-2.04)$ & 0.452 \\
\hline \multicolumn{7}{|c|}{ Microvascular invasion } \\
\hline$(+) \operatorname{vs}(-)$ & $1.12(0.77-1.63)$ & 0.548 & $1.55(0.90-2.67)$ & 0.111 & $1.42(0.10-2.02)$ & 0.051 \\
\hline \multicolumn{7}{|c|}{ Major portal vein invasion } \\
\hline$(+) \mathrm{vs}(-)$ & $0.90(0.44-1.81)$ & 0.760 & $1.73(0.81-3.68)$ & 0.156 & $0.76(0.37-1.55)$ & 0.758 \\
\hline \multicolumn{7}{|c|}{ Intrahepatic metastasis } \\
\hline$(+) \mathrm{vs}(-)$ & $5.21(3.68-7.38)$ & $<0.001$ & $4.78(3.08-7.41)$ & $<0.001$ & $3.97(2.69-5.85)$ & $<0.001$ \\
\hline \multicolumn{7}{|c|}{ Multicentric occurrence } \\
\hline$(+) \mathrm{vs}(-)$ & $1.94(1.03-3.62)$ & 0.039 & & & $1.54(0.82-2.90)$ & 0.183 \\
\hline \multicolumn{7}{|l|}{ AJCC T-stage } \\
\hline 3,4 vs 1,2 & $1.03(0.58-1.82)$ & 0.918 & $1.39(0.62-3.13)$ & 0.423 & $1.49(0.86-2.60)$ & 0.156 \\
\hline \multicolumn{7}{|l|}{ BCLC stage } \\
\hline B,C vs 0, A & $0.90(0.60-1.36)$ & 0.622 & $1.11(0.62-1.98)$ & 0.729 & $1.16(0.56-2.40)$ & 0.691 \\
\hline \multicolumn{7}{|c|}{ Albumin level, $\mathrm{g} / \mathrm{dL}$} \\
\hline$\leq 3.5 \mathrm{vs}>3.5$ & $1.32(0.77-2.23)$ & 0.311 & $1.42(0.70-2.87)$ & 0.335 & $1.30(0.77-2.20)$ & 0.32 \\
\hline \multicolumn{7}{|l|}{ ALBI grade } \\
\hline 2 vs 1 & $1.41(1.02-1.94)$ & 0.038 & $1.41(0.92-2.16)$ & 0.117 & $1.47(1.08-2.02)$ & 0.015 \\
\hline \multicolumn{7}{|l|}{ AFP level, ng/mL } \\
\hline$>200$ vs $\leq 200$ & $1.08(0.79-1.49)$ & 0.628 & $1.10(0.70-1.73)$ & 0.688 & $1.19(0.88-1.62)$ & 0.26 \\
\hline \multicolumn{7}{|l|}{ Etiology } \\
\hline Viral vs Non-viral & $1.01(0.86-1.20)$ & 0.869 & $0.92(0.72-1.16)$ & 0.477 & $0.99(0.84-1.65)$ & 0.887 \\
\hline \multicolumn{7}{|l|}{ Liver cirrhosis } \\
\hline$(+)$ vs $(-)$ & $1.32(0.97-1.79)$ & 0.078 & & & $1.29(0.96-1.74)$ & 0.096 \\
\hline \multicolumn{7}{|l|}{ miR122 expression } \\
\hline Low vs High & $1.89(1.16-3.07)$ & 0.010 & $2.14(1.05-4.36)$ & 0.036 & $2.17(1.34-3.52)$ & 0.002 \\
\hline
\end{tabular}

Table 6. Multivariate analysis of probable prognostic factors in intrahepatic recurrence- free survival, distant metastasis- free survival, and recurrence free survival. AJCC, American Joint Committee on Cancer; BCLC, Barcelona Clinic Liver Cancer; ALBI, Albumin-Bilirubin; AFP, $\alpha$-fetoprotein.

differentiation, microvascular invasion, major portal vein invasion, intrahepatic metastasis, multicentric occurrence, and non-tumor liver pathology, were reviewed by two liver pathologists (S.Y.H. and C.-K.P.). Tumor differentiation was determined according to the criteria of Edmondson and Steiner ${ }^{41}$. Intrahepatic metastasis and multicentric occurrence were distinguished according to the criteria of the Liver Cancer Study Group of Japan ${ }^{42}$. Patients were staged according to the AJCC staging system ${ }^{43}$ and BCLC staging classification ${ }^{44}$.

During the follow-up period, patients underwent computed tomography (CT) with measurement of serum AFP every 2 or 3 months postoperatively. Patients with suspicious imaging findings and/or continuously elevated AFP levels were further evaluated with PET-CT and/or MRI. The median follow-up period was 119.8 months (range 14-151.4 months) for survival. Recurrence was generally diagnosed using radiologic examinations with no histologic confirmation. The site of first recurrence was classified as intrahepatic recurrence (IHR) or distant metastases (DM). IHR is defined as recurrence occurring anywhere inside the entire liver. Intrahepatic HCC recurrence within the first two years after curative resection is mainly due to intrahepatic metastasis, whereas late recurrence usually results from multicentric disease ${ }^{45}$. Using 2 years as a cutoff, intrahepatic tumor recurrence was classified as either early or late ${ }^{46}$. All other sites of recurrence were defined as DM. The duration of IHR-free survival (IHRFS), DM-free survival (DMFS), and recurrence-free survival (RFS) was calculated from the date of surgical resection to the date of each event or the last day of follow-up.

RNA extraction and quantitative Reverse-Transcriptase Polymerase Chain Reaction (qRT-PCR). Total RNA was isolated from sliced tissue samples using the RNeasy Plus Mini Kit (Qiagen, Hilden, Germany). RT-PCR was conducted using the High Capacity cDNA Reverse Transcription Kit (Applied Biosystems) according to the manufacturer's instructions. Quantitation of miR-122 was performed using standard reagents from Applied Biosystems (TaqMan ${ }^{\circledR}$ MicroRNA Assays: miR-122: 002245). RNU6B was used as an endogenous control. Reverse transcription was performed with $25 \mathrm{ng}$ of total RNA using the TaqMan ${ }^{\circledR}$ primers from MicroRNA Assays and the TaqMan ${ }^{\circledR}$ MicroRNA Reverse Transcription Kit (4366596, Life Technologies, Carlsbad). PCR was performed in an ABI PRISM 7500HT Fast Real-time PCR with TaqMan ${ }^{\circledR}$ Universal PCR Master Mix, No AmpErase UNG (Life Technologies) according to the manufacturer's protocol. The PCR conditions were as follows: $95^{\circ} \mathrm{C}$ for $10 \mathrm{~min}$, followed by 40 cycles of amplification at $95^{\circ} \mathrm{C}$ for $15 \mathrm{~s}$ and $60^{\circ} \mathrm{C}$ for $1 \mathrm{~min}$ on the $\mathrm{ABI}$ 
PRISM 7500HT Fast Real-time PCR system (Applied Biosystems). The threshold cycle (Ct), which is the fractional cycle number at which the amount of amplified target reaches a fixed threshold, was determined. Relative changes in gene expression were measured using the $2-\Delta \Delta \mathrm{Ct}(\Delta \Delta \mathrm{Ct}=\Delta \mathrm{Cttarget}$ gene $-\Delta \mathrm{CtGAPDH})$ method. Each reaction was carried out in triplicate technical replicates. Ct values were calculated for each replicate and averaged.

To generate standard curves for genotype identification assays, 10-fold serial dilution containing a HCC190 sample was analyzed in three independent runs by RT-qPCR. In order to take into account the diagnostic context of the sample matrix, each dilution was analyzed in three independent runs by RT-qPCR. The log dilution series of was tested in triplicates in each run. Standard curves for each assay were generated by plotting threshold cycle $(\mathrm{Ct})$ values per three replicates per standard dilution versus the log concentration. Amplification efficiency, intra-assay and inter-assay variation were determined using 10-fold dilutions of one sample (No. 178). An efficiency of 1 corresponds to $100 \%$ amplification efficiency. The coefficient of determination $\left(\mathrm{R}^{2}\right)$ was assessed and considered to be suitable when it was not lower than 0.98 in a single run.

Statistical analysis. The cut-off value of miR-122 expression for RFS was determined using the log-rank test and the minimum p-value approach. The estimated cutoff value was internally validated using 1,000 bootstrap samples generated from the study data with replacement. $P$-values $<0.05$ were considered statistically significant in two-tailed tests.

The correlation between miR-122 expression and other clinicopathologic variables was evaluated using the chi-square test or Fisher's exact test. Univariate analysis was performed using the Cox proportional hazard model or the time-dependent Cox model according to satisfaction of the proportional hazard assumption for each variable. The proportional hazard assumption was confirmed using the correlation between partial residuals from the estimated Cox proportional hazard model and time to the event. Variables with $P<0.2$ in univariate analysis were included in the multivariate analysis. Multivariate analysis was conducted using a time-dependent Cox model because some variables included in the multivariate analysis failed to adhere to the proportional hazard assumption. Multicollinearity was identified by a Variance Inflation Factor (VIF) $>10.0$. Some of those variables were excluded from the multivariate analysis. Statistical analyses were performed using SAS version 9.4 (SAS Institute, Cary, NC) and SPSS software (SPSS Inc., Chicago, IL, USA). Variable risk was expressed as a hazard ratio (HR) with corresponding $95 \%$ confidence interval (CI).

\section{References}

1. El-Serag, H. B. \& Rudolph, K. L. Hepatocellular carcinoma: epidemiology and molecular carcinogenesis. Gastroenterology 132, 2557-2576, https://doi.org/10.1053/j.gastro.2007.04.061 (2007).

2. Llovet, J. M., Schwartz, M. \& Mazzaferro, V. Resection and liver transplantation for hepatocellular carcinoma. Semin Liver Dis 25, 181-200, https://doi.org/10.1055/s-2005-871198 (2005).

3. Qin, L. X. \& Tang, Z. Y. Recent progress in predictive biomarkers for metastatic recurrence of human hepatocellular carcinoma: a review of the literature. J Cancer Res Clin Oncol 130, 497-513, https://doi.org/10.1007/s00432-004-0572-9 (2004).

4. Chang, J. et al. miR-122, a mammalian liver-specific microRNA, is processed from hcr mRNA and may downregulate the high affinity cationic amino acid transporter CAT-1. RNA Biol 1, 106-113 (2004).

5. Lagos-Quintana, M. et al. Identification of tissue-specific microRNAs from mouse. Curr Biol 12, 735-739 (2002).

6. Landgraf, P. et al. A mammalian microRNA expression atlas based on small RNA library sequencing. Cell 129, 1401-1414, https:// doi.org/10.1016/j.cell.2007.04.040 (2007).

7. Wienholds, E. et al. MicroRNA expression in zebrafish embryonic development. Science 309, 310-311, https://doi.org/10.1126/ science.1114519 (2005).

8. Thakral, S. \& Ghoshal, K. miR-122 is a unique molecule with great potential in diagnosis, prognosis of liver disease, and therapy both as miRNA mimic and antimir. Curr Gene Ther 15, 142-150 (2015).

9. Lanford, R. E. et al. Therapeutic silencing of microRNA-122 in primates with chronic hepatitis C virus infection. Science 327, 198-201, https://doi.org/10.1126/science.1178178 (2010).

10. Hsu, S. H. et al. Essential metabolic, anti-inflammatory, and anti-tumorigenic functions of miR-122 in liver. J Clin Invest 122, 2871-2883, https://doi.org/10.1172/JCI63539 (2012)

11. Esau, C. et al. miR-122 regulation of lipid metabolism revealed by in vivo antisense targeting. Cell Metab 3, 87-98, https://doi. org/10.1016/j.cmet.2006.01.005 (2006).

12. Castoldi, M. et al. The liver-specific microRNA miR-122 controls systemic iron homeostasis in mice. J Clin Invest 121, 1386-1396, https://doi.org/10.1172/JCI44883 (2011)

13. Lin, C. J., Gong, H. Y., Tseng, H. C., Wang, W. L. \& Wu, J. L. miR-122 targets an anti-apoptotic gene, Bcl-w, in human hepatocellular carcinoma cell lines. Biochem Biophys Res Commun 375, 315-320, https://doi.org/10.1016/j.bbrc.2008.07.154 (2008).

14. Ladeiro, Y. et al. MicroRNA profiling in hepatocellular tumors is associated with clinical features and oncogene/tumor suppressor gene mutations. Hepatology 47, 1955-1963, https://doi.org/10.1002/hep.22256 (2008).

15. Kutay, H. et al. Downregulation of miR-122 in the rodent and human hepatocellular carcinomas. J Cell Biochem 99, 671-678, https:// doi.org/10.1002/jcb.20982 (2006).

16. Gramantieri, L. et al. Cyclin G1 is a target of miR-122a, a microRNA frequently down-regulated in human hepatocellular carcinoma. Cancer Res 67, 6092-6099, https://doi.org/10.1158/0008-5472.CAN-06-4607 (2007).

17. Girard, M., Jacquemin, E., Munnich, A., Lyonnet, S. \& Henrion-Caude, A. miR-122, a paradigm for the role of microRNAs in the liver. J Hepatol 48, 648-656, https://doi.org/10.1016/j.jhep.2008.01.019 (2008)

18. Budhu, A. et al. Identification of metastasis-related microRNAs in hepatocellular carcinoma. Hepatology 47, 897-907, https://doi. org/10.1002/hep.22160 (2008).

19. Braconi, C. \& Patel, T. MicroRNA expression profiling: a molecular tool for defining the phenotype of hepatocellular tumors. Hepatology 47, 1807-1809, https://doi.org/10.1002/hep.22326 (2008).

20. Murakami, Y. et al. Comprehensive analysis of microRNA expression patterns in hepatocellular carcinoma and non-tumorous tissues. Oncogene 25, 2537-2545, https://doi.org/10.1038/sj.onc.1209283 (2006).

21. Tsai, W. C. et al. MicroRNA-122, a tumor suppressor microRNA that regulates intrahepatic metastasis of hepatocellular carcinoma. Hepatology 49, 1571-1582, https://doi.org/10.1002/hep.22806 (2009).

22. Tsai, W. C. et al. MicroRNA-122 plays a critical role in liver homeostasis and hepatocarcinogenesis. J Clin Invest 122, 2884-2897, https://doi.org/10.1172/JCI63455 (2012).

23. Xu, Q. et al. MicroRNA-122 affects cell aggressiveness and apoptosis by targeting PKM2 in human hepatocellular carcinoma. Oncol Rep 34, 2054-2064, https://doi.org/10.3892/or.2015.4175 (2015). 
24. Wu, Q. et al. Decreased expression of hepatocyte nuclear factor 4alpha (Hnf4alpha)/microRNA-122 (miR-122) axis in hepatitis B virus-associated hepatocellular carcinoma enhances potential oncogenic GALNT10 protein activity. J Biol Chem 290, 1170-1185, https://doi.org/10.1074/jbc.M114.601203 (2015).

25. Jin, Y., Wang, J., Han, J., Luo, D. \& Sun, Z. MiR-122 inhibits epithelial-mesenchymal transition in hepatocellular carcinoma by targeting Snail1 and Snail2 and suppressing WNT/beta-cadherin signaling pathway. Exp Cell Res 360, 210-217, https://doi. org/10.1016/j.yexcr.2017.09.010 (2017).

26. Coulouarn, C., Factor, V. M., Andersen, J. B., Durkin, M. E. \& Thorgeirsson, S. S. Loss of miR-122 expression in liver cancer correlates with suppression of the hepatic phenotype and gain of metastatic properties. Oncogene 28, 3526-3536, https://doi. org/10.1038/onc.2009.211 (2009).

27. Hsu, S. H. et al. Cationic lipid nanoparticles for therapeutic delivery of siRNA and miRNA to murine liver tumor. Nanomedicine 9 , 1169-1180, https://doi.org/10.1016/j.nano.2013.05.007 (2013).

28. Lo, A., Lin, C. T. \& Wu, H. C. Hepatocellular carcinoma cell-specific peptide ligand for targeted drug delivery. Mol Cancer Ther 7 , 579-589, https://doi.org/10.1158/1535-7163.MCT-07-2359 (2008).

29. Zhang, M. et al. Lactosylated gramicidin-based lipid nanoparticles (Lac-GLN) for targeted delivery of anti-miR-155 to hepatocellular carcinoma. J Control Release 168, 251-261, https://doi.org/10.1016/j.jconrel.2013.03.020 (2013).

30. Zhang, Y., Li, Y., Jiang, W., Li, Q. \& Lan, Y. The clinical significance of microRNA-122 in predicting the prognosis of patients with hepatocellular carcinoma: A meta-analysis validated by the Cancer Genome Atlas dataset. Medicine (Baltimore) 98, e14810, https:// doi.org/10.1097/MD.0000000000014810 (2019).

31. Qiao, D. D. et al. Expression of microRNA-122 and microRNA-22 in HBV-related liver cancer and the correlation with clinical features. Eur Rev Med Pharmacol Sci 21, 742-747 (2017).

32. Suehiro, T. et al. Serum exosomal microRNA-122 and microRNA-21 as predictive biomarkers in transarterial chemoembolizationtreated hepatocellular carcinoma patients. Oncol Lett 16, 3267-3273, https://doi.org/10.3892/ol.2018.8991 (2018).

33. Xue, X., Zhao, Y., Wang, X., Qin, L. \& Hu, R. Development and validation of serum exosomal microRNAs as diagnostic and prognostic biomarkers for hepatocellular carcinoma. J Cell Biochem 120, 135-142, https://doi.org/10.1002/jcb.27165 (2019).

34. Kishikawa, T. et al. Decreased miR122 in hepatocellular carcinoma leads to chemoresistance with increased arginine. Oncotarget 6, 8339-8352, https://doi.org/10.18632/oncotarget.3234 (2015).

35. Agostini, M. \& Knight, R. A. miR-34: from bench to bedside. Oncotarget 5, 872-881, https://doi.org/10.18632/oncotarget.1825 (2014).

36. Yang, F. et al. Modulation of the unfolded protein response is the core of microRNA-122-involved sensitivity to chemotherapy in hepatocellular carcinoma. Neoplasia 13, 590-600 (2011).

37. Xu, Y. et al. MicroRNA-122 sensitizes HCC cancer cells to adriamycin and vincristine through modulating expression of MDR and inducing cell cycle arrest. Cancer Lett 310, 160-169, https://doi.org/10.1016/j.canlet.2011.06.027 (2011).

38. Wang, B. et al. Role of microRNA-155 at early stages of hepatocarcinogenesis induced by choline-deficient and amino acid-defined diet in C57BL/6 mice. Hepatology 50, 1152-1161, https://doi.org/10.1002/hep.23100 (2009).

39. Bai, S. et al. MicroRNA-122 inhibits tumorigenic properties of hepatocellular carcinoma cells and sensitizes these cells to sorafenib. J Biol Chem 284, 32015-32027, https://doi.org/10.1074/jbc.M109.016774 (2009).

40. Yu, J. I. et al. Clinical importance of TERT overexpression in hepatocellular carcinoma treated with curative surgical resection in HBV endemic area. Sci Rep 7, 12258, https://doi.org/10.1038/s41598-017-12469-2 (2017).

41. Edmondson, H. \& Steiner, P. Primary carcinoma of the liver: a study of 100 cases among 48,900 necropsies. Cancer 7, 462-503 (1954).

42. Liver Cancer Study Group of Japan. The general rules for the clinical and pathological study of primary liver cancer. 2nd edn (2003).

43. Edge, S. et al. AJCC cancer staging manual. 7th edn (Springer, 2010).

44. Llovet, J. M., Bru, C. \& Bruix, J. Prognosis of hepatocellular carcinoma: the BCLC staging classification. Seminars in liver disease 19, 329-338, https://doi.org/10.1055/s-2007-1007122 (1999).

45. Shimada, M. et al. Characteristics of multicentric hepatocellular carcinomas: comparison with intrahepatic metastasis. World J Surg 25, 991-995 (2001).

46. Imamura, H. et al. Risk factors contributing to early and late phase intrahepatic recurrence of hepatocellular carcinoma after hepatectomy. J Hepatol 38, 200-207 (2003).

\section{Acknowledgements}

This research was supported by a Samsung Medical Center grant (GFO02160081 and OTA1603741).

\section{Author Contributions}

Conception and design: S.Y. Ha, J.I. Yu, C. Choi, H.C. Park, C.K. Park. Development of methodology: S.Y. Ha, J.I. Yu, C. Choi, S.Y. Kang, S. Kim, M. Kim, H.C. Park, C.K. Park. Acquisition of data: S.Y. Ha, J.I. Yu, C. Choi, H.C. Park, C.K. Park, J.W. Joh, S.W. Paik. Analysis and interpretation of data (e.g., statistical analysis, biostatistics, computational analysis): S.Y. Ha, J.I. Yu, C. Choi, H.C. Park, C.K. Park. Writing: S.Y. Ha, J.I. Yu, C. Choi, H.C. Park, C.K. Park. Review, and/or revision of the manuscript: all authors.

\section{Additional Information}

Supplementary information accompanies this paper at https://doi.org/10.1038/s41598-019-50594-2.

Competing Interests: The authors declare no competing interests.

Publisher's note Springer Nature remains neutral with regard to jurisdictional claims in published maps and institutional affiliations.

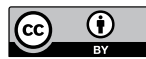

Open Access This article is licensed under a Creative Commons Attribution 4.0 International License, which permits use, sharing, adaptation, distribution and reproduction in any medium or format, as long as you give appropriate credit to the original author(s) and the source, provide a link to the Creative Commons license, and indicate if changes were made. The images or other third party material in this article are included in the article's Creative Commons license, unless indicated otherwise in a credit line to the material. If material is not included in the article's Creative Commons license and your intended use is not permitted by statutory regulation or exceeds the permitted use, you will need to obtain permission directly from the copyright holder. To view a copy of this license, visit http://creativecommons.org/licenses/by/4.0/.

(C) The Author(s) 2019 